\title{
Static output feedback stabilization of discrete time linear time invariant systems based on approximate dynamic programming
}

Transactions of the Institute of Measurement and Control 2020, Vol. 42(16) 3168-3182 (c) The Author(s) 2020

Article reuse guidelines: sagepub.com/journals-permissions DOI: 10.1 | $77 / 014233$ |22094307| journals.sagepub.com/home/tim

@SAGE

\author{
Okan Demir (D) and Hitay Özbay
}

\begin{abstract}
This study proposes a method for the static output feedback (SOF) stabilization of discrete time linear time invariant (LTI) systems by using a low number of sensors. The problem is investigated in two parts. First, the optimal sensor placement is formulated as a quadratic mixed integer problem that minimizes the required input energy to steer the output to a desired value. Then, the SOF stabilization, which is one of the most fundamental problems in the control research, is investigated. The SOF gain is calculated as a projected solution of the Hamilton-Jacobi-Bellman (HJB) equation for discrete time LTI system. The proposed method is compared with several examples from the literature.
\end{abstract}

\section{Keywords}

Static output feedback, optimal sensor placement, approximate dynamic programming

\section{Introduction}

Spatially distributed dynamical systems, such as flexible structures (Halevi and Wagner-Nachshoni, 2006), diffusion (Garvie, 2007), biological systems (Turing, 1990; Vicsek and Zafeiris, 2012), are modeled by high dimensional state space equations in order to capture all essential properties of their physical character. When considered in the control systems perspective, this leads to high dimensional controllers that require a large number of sensors. The optimal sensor placement problem is substantial to improve observability and controllability of the system (Shaker and Tahavori, 2013). It changes the location of the zeros of system on the complex plane which may limit the closed-loop performance. Sensor locations also affect the cost of control implementation (van de Wal and de Jager, 2001; Zhang and Morris, 2018). Present day applications of the control of large scale systems extend over complex systems like computer networks, power grids and biological networks (Klickstein et al., 2017; Liu et al., 2011). Improving the controllability and observability of large scale systems are important to define better control laws in terms of performance, robustness and feasibility of the physical implementation. For this respect, there are quantitative approaches in the literature based on improving the system gramians (Belabbas and Chen, 2018; Bender, 1987; Casadei, 2018; Klickstein et al., 2017; Marx et al., 2002; Shaker and Tahavori, 2013; Summers and Lygeros, 2014; Summers et al., 2016; van de Wal and de Jager, 2001). On the other hand, problem can be analyzed by using the structural properties of dynamical system (Chen et al., 2019; Belabbas, 2016;
Klickstein et al., 2017; Liu et al., 2011; Müller and Schuppert, 2011).

For linear time invariant (LTI) systems, the controllability and observability matrices and gramians provide quantitative measures. The observability/controllability condition imposed by gramians can be improved by an optimal selection of the sensor/actuator locations. The matrix norms of observability/ controllability gramians are reliable measures to determine the sensor and actuator locations (Summers and Lygeros, 2014). The input and output matrices must be designed to maximize the controllability and observability gramians. In the first part of this paper, for a fixed input matrix $B$ (fixed actuator configuration) a low dimensional optimal output matrix $C$ (sensor placement) is investigated. The considered systems are not supposedly stable. Hence, a discrete time counterpart of the generalized gramian calculation in Zhou et al. (1999) is developed. The considered systems are assumed to be output controllable in the sense that is defined in Klickstein et al. (2017). The optimal sensor selection is formulated as a norm maximization problem for the "output controllability gramian" matrix. It is also shown that outputs chosen by this method can be steered to a desired value with a minimum amount of input energy. It can be said that the

Electrical and Electronics Engineering, Bilkent University, Turkey

Corresponding author:

Okan Demir, Department of Electrical and Electronics Engineering, Bilkent University, Çankaya, Ankara, Çankaya 06800, Turkey.

Email: demir@ee.bilkent.edu.tr 
smaller input energy requirement means the larger influence of the input on the output (Çela et al., 2018). The intuition behind using the sensors chosen in this way is to obtain relatively smaller static output feedback (SOF) gains having a larger effect on the output of the next time step.

Once the output matrix is determined as above, the second part of the paper discusses the computation of a stabilizing SOF gain. For many systems full state variable information may not be available or it may be costly to place many sensors. If the system is observable, an observer and state feedback configuration can be implemented. But, this will lead to high dimensional controllers for large scale systems. Stabilization by SOF is preferred because of its simplicity. However, SOF problem is known to be $N P$-hard (Toker and Özbay, 1995) because of the non-convexity of the problem (Sadabadi and Peaucelle, 2016). In fact, not all plants can be stabilized by a static output feedback, a necessary condition for the existence of SOF controller is that the plant satisfies the parity interlacing property (PIP), so that it is stabilizable by a stable controller. Moreover, even if the plant satisfies the PIP, depending on the location of non-minimum phase zeros and unstable poles, the order of the stabilizing stable controller may have to be high (Smith and Sondergeld, 1986). For recent work and further references on stable controller design, see Yücesoy and Özbay (2019).

There is a vast literature on the numerical solution of the SOF problem for both continuous and discrete time LTI systems (Bara and Boutayeb, 2005; Gadewadikar and Lewis, 2006; Garcia et al., 2001; Palacios-Quionero, et al., 2012, 2014; Sadabadi and Peaucelle, 2016). In Garcia et al. (2001), the SOF gain is directly obtained from the system matrices, which does not allow adding constraint on the robustness and performance. Several approaches formulate the SOF stabilization as a linear matrix inequality (LMI) problem. In Bara and Boutayeb (2005), the SOF gain is found from the solutions of two consecutive LMI problems when a proper realization of the state space model is used. There are methods those obtain the SOF gain as the solution of a single LMI (Crusius and Trofino, 1999; Palacios-Quionero et al., 2012, 2014). In these methods, robustness and performance conditions are formulated by adding extra constraints on the LMIs. For our proposed method, the calculated SOF gain leads to a similar quadratic cost as the Linear Quadratic Regulator (LQR) problem with a larger cost function weight on the states. It can be said that it has similar performance characteristics in $L^{2}$-norm measure.

There are iterative approaches using sequential solutions of the Riccati equations those lack convergence guarantee (Gadewadikar and Lewis, 2006; Gadewadikar et al., 2007; Rosinová et al., 2003). This study proposes another iterative approach based on approximate dynamic programming (ADP). The SOF gain is calculated as a projected solution of the well-known Hamilton-Bellman-Jacobi (HJB) iterations for the discrete time LTI systems (Bertsekas, 1995). Nevertheless, solutions lack convergence guarantee and are dependent on the system's realization similar to the counterparts in the literature. Despite the inevitable numerical intractability of the problem, a necessary condition for the system matrices can be defined. Promising results are obtained for the balanced form of example models.
The results are compared with some examples from the literature (Bara and Boutayeb, 2005; Gadewadikar et al., 2007; Garcia et al., 2001). A significant improvement in the results is observed according to the robustness metrics and the spectral radius of the closed loop system matrix. Also, these characteristics can be easily adjusted by changing the cost function weights. Furthermore, its applicability is demonstrated on a truncated version of the simply supported flexible beam model and a large scale biological network (Edelstein-Keshet, 2005; Hiramoto et al., 2000).

The paper is organized as follows. In Section 1, the problem is defined formally. In Section 2, optimal sensor placement for unstable systems is investigated. Approximate solution of the LQR problem is discussed in Section 3. The proposed method is demonstrated on several examples in Section 4. Lastly, there are remarks and discussions on the results in Section 5.

\section{Problem formulation}

The systems considered in this study are discrete time linear time invariant (LTI) systems given by the state space representation

$$
\begin{gathered}
x_{t+1}=A x_{t}+B u_{t} \\
y_{t}=C x_{t}+D u_{t},
\end{gathered}
$$

where $x \in \mathbb{R}^{n}$ is the state vector, $u \in \mathbb{R}^{m}$ is the input and $y \in \mathbb{R}^{q}$ is the output of system. $A, B, C, D$ are constant matrices of appropriate dimensions and we assume $D=0$. It is also assumed that the pair $(A, B)$ is stabilizable and $(A, C)$ is detectable. The output matrix $C$ is of rank $q$ and is expressed as

$$
C=\operatorname{diag}\left(\left\{\alpha_{i}\right\}_{i=1}^{r}\right) \tilde{C},
$$

where the number of rows of $\tilde{C}$ is assumed to be $r \geqslant q$, and it captures all available sensors sites at various different possible locations (each sensor corresponds to a row of $\tilde{C}$ ), and $\alpha_{i} \in\{0,1\} \forall i=1, \cdots, r$ (with $r-q$ of them being zero). We are interested in using a low number of sensors ( $q$ of them).

Problem definition: Given the system model $(A, B, \tilde{C})$, which is not necessarily stable, $\gamma>0$, symmetric matrices $Q \geqslant 0$ and $R>0$, find $K$ and $\alpha_{1}, \cdots, \alpha_{r}$ by solving

$$
\begin{gathered}
\min _{K, \alpha_{i}}\left(\gamma q+\sum_{t=0}^{\infty} x_{t}^{T} Q x_{t}+u_{t}^{T} R u_{t}\right) \\
\text { subject to } x_{t+1}=A x_{t}+B u_{t} \\
u_{t}=K y_{t}=K \operatorname{diag}\left(\left\{\alpha_{i}\right\}_{i=1}^{r}\right) \tilde{C} x_{t} \\
\sum_{i=1}^{r} \alpha_{i}=q \in\{1, \cdots, r\}, \alpha_{i} \in\{0,1\} .
\end{gathered}
$$

Direct optimal solution of this problem is difficult. For this reason we separate it into two optimization problems. First, $\alpha_{i}$ values are found to construct the output matrix $C$ which is optimal in terms of minimizing the required input energy to steer the output to a desired value. Then, a stabilizing SOF 
gain $K$ is calculated for the output matrix $C$ obtained from the first problem.

Notation used in the paper is standard. In particular, for a system described in the state space form given by (1) and (2) we may also use the compact notation

$$
\left\lceil\frac{A}{C} \mid \frac{B}{D}\right]
$$

to represent the same system, as in Zhou et al. (1996).

\section{Sensor selection problem}

Controllability gramian of a discrete time LTI system is defined as

$$
W_{c}=\sum_{t=0}^{\infty} A^{t} B B^{T}\left(A^{T}\right)^{t}
$$

where the gramian $W_{c}$ is a symmetric non-negative definite matrix. Its norm $\left\|W_{c}\right\|$ is a measure for the required input energy to steer state vector $x$ from $x_{0}$ to $x_{t}$. A larger $\left\|W_{c}\right\|$ means that less energy is required and the system has a higher degree of controllability (Marx et al., 2002; Shaker and Tahavori, 2013; Summers and Lygeros, 2014; van de Wal and de Jager, 2001).

\section{The gramians of unstable discrete time $L T I$ systems}

Equation (4) is undefined unless $A$ has all eigenvalues inside the unit circle. However, a discrete time counterpart of the generalized gramian calculation algorithm for unstable continuous time LTI systems in Zhou et al. (1999) can be developed as follows.

Assuming $(A, B)$ is stabilizable and $A$ has no eigenvalues on the unit circle, transform the unstable system $G(z)=\tilde{C}(z I-A)^{-1} B$ into its co-prime factorized form $G(z)=N(z) M(z)^{-1}$ where $N(z)$ and $M(z)$ are stable and $M(z)$ is an inner transfer function (Zhou et al., 1996). The right coprime factorization of $G(z)$ in the state space form can be given by

$$
\left[\begin{array}{c|c}
A+B F & B \tilde{R}^{-1 / 2} \\
\hline F & \tilde{R}^{-1 / 2} \\
\tilde{C} & 0
\end{array}\right],
$$

where $\tilde{R}=I+B^{T} S B, F=-\tilde{R}^{-1} B^{T} S A$ and $S=S^{T} \geqslant 0$ is the stabilizing solution of

$$
S=A^{T} S\left(I+B B^{T} S\right)^{-1} A .
$$

The solutions $S$ of (5) also satisfies the discrete time algebraic Riccati equation (DARE) (Lancaster and Rodman, 1995)

$$
S=A^{T} S A-A^{T} S B\left(I+B^{T} S B\right)^{-1} B^{T} S A .
$$

Then, the generalized controllability gramian is a symmetric matrix $W_{c} \geqslant 0$ that satisfies the following Lyapunov equation

$$
(A+B F) W_{c}(A+B F)^{T}+B \tilde{R}^{-1} B^{T}=W_{c} .
$$

Calculating the generalized observability gramian is straight forward for the dual system $\left(A^{T}, C^{T}\right)$.

\section{Output controllability}

For the system given by $(A, B, C)$, if an input sequence $u_{t}$ can be found that steers the system output from $y_{0}$ to a desired $y_{f}$ in finite time, it can be said that $(A, B, C)$ is output controllable, (Klickstein et al., 2017). Output controllability can be defined as a matrix rank condition

$$
\text { If } \operatorname{rank}(C)=\operatorname{rank}\left(C\left[\begin{array}{llll}
B & A B & \cdots & A^{n-1} B
\end{array}\right]\right),
$$

the system is output controllable. Similarly, the output controllability gramian can be defined as (Casadei, 2018; Klickstein et al., 2017)

$$
Y_{c}=C W_{c} C^{T},
$$

where $W_{c}$ is the generalized controllability gramian. The norm of $Y_{c}$ gives a measure about ease of steering the output of the system to a desired value if the system is output controllable. Output controllability condition (8) implies non-singularity of the output controllability gramian $Y_{c}$ (Klickstein et al., 2017) (discrete time version can be found in Rugh (1996).

Lemma 1: For the unstable discrete time LTI system defined by $(A, B, C)$, define

$$
\begin{aligned}
Y_{c}(t) & =C \sum_{\tau=0}^{t-1}(A+B F)^{t-\tau-1} B\left(I+B^{T} S B\right)^{-1} B^{T} \\
& \times\left((A+B F)^{T}\right)^{t-\tau-1} C^{T}
\end{aligned}
$$

where $S$ is given in (6) and $F=-\left(I+B^{T} S B\right)^{-1} B^{T} S A$. Then, the minimum energy required to steer the output $y_{0}=0$ to a desired final value $y_{t_{f}}=y_{f}$ is given by

$$
J=\frac{1}{2} y_{f}^{T} Y_{c}\left(t_{f}\right)^{-1} y_{f}
$$

where $J$ is also the solution of

$$
\begin{gathered}
\min _{u_{t}} J=\frac{1}{2} x_{t_{f}}^{T} S x_{t_{f}}+\frac{1}{2} \sum_{t=0}^{t_{f}-1} u_{t}^{T} u_{t} \\
\text { s.t. } x_{t+1}=A x_{t}+B u_{t}, y_{t}=C x_{t} \\
y_{f}=y_{t_{f}}=C x_{t_{f}} .
\end{gathered}
$$

Proof: Proof can be found in the Appendix. 
Remark 1: Note that

$$
\begin{aligned}
Y_{c}(t) & =C \sum_{\tau=0}^{t-1}(A+B F)^{t-\tau-1} B\left(I+B^{T} S B\right)^{-1} B^{T} \\
& \times\left((A+B F)^{T}\right)^{t-\tau-1} C^{T} \\
& =C \sum_{\tau=0}^{t-1}(A+B F)^{\tau} B\left(I+B^{T} S B\right)^{-1} B^{T} \\
& \times\left((A+B F)^{T}\right)^{\tau} C^{T} \\
& =C W_{c}(t) C^{T},
\end{aligned}
$$

where $W_{c}(t)$ is the generalized controllability gramian in (7) and $W_{c}(t) \rightarrow W_{c}$ as $t \rightarrow \infty$.

Now, formulate the problem as maximizing the Frobenius norm of $Y_{c}$ with respect to $\alpha_{i}$ of (3) by writing it as a quadratic problem

$$
\begin{aligned}
& \text { Define } \alpha:=\left[\alpha_{1}, \cdots, \alpha_{r}\right]^{T}, \\
& \max _{\alpha} \alpha^{T} H \alpha, \\
& \text { s.t } \sum_{i=1}^{r} \alpha_{i}=q,
\end{aligned}
$$

$$
\text { given } q \in\{1, \cdots, r\}, \alpha_{i} \in\{0,1\} \text {, }
$$

where the elements of $H_{i j}$ is the magnitude square of the elements of $Y_{c}$. This problem can be solved by mixed integer programming tools. In this study, solutions are obtained from SCIP software (Gamrath et al., 2020) by solving for all $q=\{1, \cdots, r\}$ to find a set of optimal $\alpha$ vectors those fulfill this directive: Choose $q$ of the outputs which are 'easier' (regarding Lemma 1) to be steered to their desired values.

\section{Static output feedback}

Now, consider the static output feedback stabilization problem where the stabilizing input is in the form of $u_{t}=K y_{t}$. The optimization problem associated with this setting is to find a SOF gain $K$ that places the eigenvalues of the closed-loop system matrix $A_{c l}=A+B K C$ inside the unit circle.

The SOF stabilization is known to be an NP-hard problem, that is, it is difficult to find a computationally efficient algorithm for its solution in complete generality (Mercado and Liu, 2001; Nemirovskii, 1993; Polyak and Shcherbakov, 2005; Toker and Özbay, 1995). However, the SOF problem is considered as an important question in the control theory and studied in many research papers (Bara and Boutayeb, 2005; Gadewadikar et al., 2007; Garcia et al., 2001; Gu, 1990; Rosinová et al., 2003; Trofino-Neto and Kučera, 1993). Furthermore, many control problems can be reduced to a SOF stabilization problem by an appropriate augmentation of the system matrices. There is neither a generally applicable way of finding a stabilizing $K$ nor determining the existence of such a $K$. In the literature, it is investigated from different aspects. In Fu (2004), SOF is formulated as a pole placement problem; the author concludes with a result that strengthens the NP-hardness assertion. Garcia et al. (2001) proposed a direct solution for the discrete time SOF stabilization by using the system matrices if the system suits some restrictive conditions. There are approaches those use LMIs derived from Lyapunov equation (Bara and Boutayeb, 2005) and iterative solution of Riccati equations (Gadewadikar et al., 2007; Rosinová et al., 2003). For a recent survey on the SOF problem, see Sadabadi and Peaucelle (2016).

In the proposed method, $K$ is found as a projected solution of the HJB equation for discrete time LTI systems. Solution is analogous to approximate dynamic programming (ADP) approach since the policy iteration step is approximated by a least squares solution (Lagoudakis and Parr, 2003). We note that balanced form of the system is advantageous to overcome the convergence issues of the dynamic programming iterations.

Definition of the finite horizon discrete time LQR problem starts with a quadratic cost $V$ given by

$$
V=x_{t_{f}}^{T} Q x_{t_{f}}+\sum_{t=0}^{t_{f}-1} x_{t}^{T} Q x_{t}+u_{t}^{T} R u_{t}
$$

which must be minimized subject to the system dynamics

$$
x_{t+1}=A x_{t}+B u_{t} .
$$

Related HJB equation can be written as (Bertsekas, 1995)

$$
\begin{gathered}
V_{t}=x_{t}^{T} Q x_{t}+u_{t}^{T} R u_{t}+x_{t+1}^{T} S_{t+1} x_{t+1} \\
=x_{t}^{T} Q x_{t}+u_{t}^{T} R u_{t}+\left(A x_{t}+B u_{t}\right)^{T} S_{t+1}\left(A x_{t}+B u_{t}\right),
\end{gathered}
$$

where $V_{t}$ is the cost at time $t$ which can be minimized by $u_{t}=-\left(R+B^{T} S_{t+1} B\right)^{-1} B^{T} S_{t+1} A x_{t}=F_{t} x_{t}$. When $u_{t}$ is substituted into (18)

$$
\begin{gathered}
V_{t}=x_{t}^{T}\left(Q+F_{t}^{T} R F_{t}+\left(A+B F_{t}\right)^{T} S_{t+1}\left(A+B F_{t}\right)\right) x_{t} \\
S_{t}=Q+F_{t}^{T} R F_{t}+\left(A+B F_{t}\right)^{T} S_{t+1}\left(A+B F_{t}\right),
\end{gathered}
$$

is obtained where $(20)$ is the value iteration step. If $(A, B)$ is stabilizable, $R=R^{T}>0, \quad Q=Q^{T} \geqslant 0$ and starting from $S_{t_{f}}=Q$, as $t \rightarrow-\infty, S_{t}$ converges to a symmetric and nonnegative definite solution $S_{t}=S$ that satisfies the discrete time algebraic Riccati equation (DARE) (Lancaster and Rodman, 1995)

$$
S=Q+F^{T} R F+(A+B F)^{T} S(A+B F)
$$

where

$$
F=-\left(R+B^{T} S B\right)^{-1} B^{T} S A
$$

is a stabilizing state feedback gain.

For the SOF case, feedback gain matrix must be structured as $F=K C$. Therefore, a SOF gain $\mathrm{K}$ and symmetric $S \geqslant 0$ must be found satisfying

$$
S=Q+C^{T} K^{T} R K C+(A+B K C)^{T} S(A+B K C)
$$

for the closed-loop stability. An optimal $F$ in this structure may not be achievable, but at each iteration of (20) a 
sub-optimal $K_{t}$ can be found by solving the following least squares problem

$$
\begin{gathered}
K_{t} C=F_{t} \\
K_{t}=F_{t} C^{T}\left(C C^{T}\right)^{-1}=F_{t} C^{\dagger},
\end{gathered}
$$

where $C$ is full row rank. Now $K_{t} C$ can be substituted into (20) instead of $F_{t}$. If $S_{t}$ converges to a $S_{t}=S$ by using the sub-optimal least squares solution, it can be said that $K=-\left(R+B^{T} S B\right)^{-1} B^{T} S A C^{\dagger}$ is a stabilizing SOF gain.

Lemma 2: Given a realization $(A, B, C)$ of an observable and controllable system and a matrix $Q=\gamma C^{T} C$, a necessary condition for the existence of a stabilizing SOF gain $K$ that satisfies

$$
S=Q+C^{T} K^{T} R K C+(A+B K C)^{T} S(A+B K C),
$$

for a symmetric $S>0$ is such that the projected system matrix $A \Pi_{\bar{c}}$ must be stable where $\Pi_{\bar{c}}=I_{n}-C^{T}\left(C C^{T}\right)^{-1} C$ is the orthogonal projection on $n u l l(C)$ and $I_{n}$ is the $n$-dimensional identity matrix.

Proof: Eigenvalue decomposition of the orthogonal projection matrix $\Pi_{c}=C^{T}\left(C C^{T}\right)^{-1} C$ is in the form of $\Pi_{c}=U_{c} \Lambda_{c} U_{c}^{T}$, where $U_{c}$ is unitary and $\Lambda_{c}=\operatorname{diag}\left(I_{r}, 0_{n-r}\right)$ where $0_{n-r}$ is an $(n-r) \times(n-r)$ matrix of zeros. Use $U_{c}$ as a similarity transformation to obtain $\tilde{A}=U_{c}^{T} A U_{c}$, $\tilde{B}=U_{c}^{T} B, \tilde{C}=C U_{c}, \tilde{Q}=\gamma \tilde{C}^{T} \tilde{C}$ and $\tilde{S}=U_{c}^{T} S U_{c}$ where $\tilde{C}$ is now in the form of $\tilde{C}=\left[\begin{array}{ll}\tilde{C}_{1} & 0\end{array}\right]$ and the corresponding projection matrix on the null space of $C$ is $\Lambda_{\bar{c}}=I_{n}-\Lambda_{c}$. Project $\tilde{S}$ by multiplying $\Lambda_{\bar{c}}$ from both sides to obtain

$$
\begin{gathered}
\Lambda_{\bar{c}} \tilde{S} \Lambda_{\bar{c}}=\Lambda_{\bar{c}} \tilde{A}^{T} \tilde{S} \tilde{A} \Lambda_{\bar{c}} \\
\tilde{S} \geqslant \Lambda_{\bar{c}} \tilde{A}^{T} \tilde{S} \tilde{A} \Lambda_{\bar{c}} \\
{\left[\begin{array}{cc}
\tilde{S}_{1} & \tilde{S}_{3} \\
\tilde{S}_{3}^{T} & \tilde{S}_{2}
\end{array}\right] \geqslant\left[\begin{array}{cc}
0 & 0 \\
\tilde{A}_{12}^{T} & \tilde{A}_{22}^{T}
\end{array}\right]\left[\begin{array}{cc}
\tilde{S}_{1} & \tilde{S}_{3} \\
\tilde{S}_{3}^{T} & \tilde{S}_{2}
\end{array}\right]\left[\begin{array}{cc}
0 & \tilde{A}_{12} \\
0 & \tilde{A}_{22}
\end{array}\right]}
\end{gathered}
$$

$\tilde{S}$ can be decomposed to

$$
\begin{aligned}
\tilde{S} & =\left[\begin{array}{cc}
\tilde{S}_{1} & \tilde{S}_{3} \\
\tilde{S}_{3}^{T} & \tilde{S}_{2}
\end{array}\right] \\
& =\left[\begin{array}{cc}
I_{r} & 0 \\
\tilde{S}_{3}^{T} \tilde{S}_{1}^{-1} & I_{n-r}
\end{array}\right]\left[\begin{array}{cc}
\tilde{S}_{1} & 0 \\
0 & \bar{S}_{2}
\end{array}\right]\left[\begin{array}{cc}
I_{r} & \tilde{S}_{1}^{-1} \tilde{S}_{3} \\
0 & I_{n-r}
\end{array}\right] \\
& =U_{p}^{T} \bar{S} U_{p},
\end{aligned}
$$

where $\tilde{S}_{1}$ and $\bar{S}_{2}=\tilde{S}_{2}-\tilde{S}_{3}^{T} \tilde{S}_{1}^{-1} \tilde{S}_{3}$ are positive definite. Then

$$
\bar{S} \geqslant\left(U_{p}^{T}\right)^{-1} \Lambda_{\bar{c}} \tilde{A}^{T} U_{p}^{T} \bar{S} U_{p} \tilde{A} \Lambda_{\bar{c}} U_{p}^{-1}
$$

$$
\begin{aligned}
{\left[\begin{array}{cc}
\tilde{S}_{1} & 0 \\
0 & \bar{S}_{2}
\end{array}\right] } & \geqslant\left[\begin{array}{cc}
0 & 0 \\
\tilde{A}_{12}^{T}+\tilde{A}_{22}^{T} \tilde{S}_{3}^{T} \tilde{S}_{1}^{-1} & \tilde{A}_{22}^{T}
\end{array}\right] \\
& \times\left[\begin{array}{cc}
\tilde{S}_{1} & 0 \\
0 & \bar{S}_{2}
\end{array}\right]\left[\begin{array}{cc}
0 & \tilde{A}_{12}+\tilde{S}_{1}^{-1} \tilde{S}_{3} \tilde{A}_{22} \\
0 & \tilde{A}_{22}
\end{array}\right],
\end{aligned}
$$

leads to

$$
\begin{aligned}
\bar{S}_{2} & \geqslant \tilde{A}_{22}^{T} \bar{S}_{2} \tilde{A}_{22} \\
& +\left(\tilde{A}_{12}+\tilde{S}_{1}^{-1} \tilde{S}_{3} \tilde{A}_{22}\right)^{T} \tilde{S}_{1}\left(\tilde{A}_{12}+\tilde{S}_{1}^{-1} \tilde{S}_{3} \tilde{A}_{22}\right) \\
& >\tilde{A}_{22}^{T} \bar{S}_{2} \tilde{A}_{22} \\
0 & >\tilde{A}_{22}^{T} \bar{S}_{2} \tilde{A}_{22}-\bar{S}_{2},
\end{aligned}
$$

which imposes stability of $\tilde{A}_{22}$ meaning that $\tilde{A} \Lambda_{\bar{c}}$ is stable. Hence, the proof can be concluded by saying $A \Pi_{\bar{c}}$ must be stable, since the similarity transformation $U_{c}$ is unitary.

Furthermore, Lemma 2 may be fulfilled for a realization of the system while being unsatisfied for an other. The convergence of $S_{t}$ highly depends on the realization. In the next section, simulation results are obtained for the balanced realization. In the balanced form, states ordered from the higher observable and controllable to the lower ones. If $C_{1} \in \mathbb{R}^{q \times q}$ in $C=\left[\begin{array}{ll}C_{1} & C_{2}\end{array}\right]$ is non-singular, there is one-toone relation between the output $y_{t}$ and the highest observable and controllable states. It can be said that $y_{t}$ holds a large amount of information about the system in the balanced form. Furthermore, it allows to neglect the states which correspond to zero Hankel Singular Values and reduce the system's dimension. Hence, using the proposed method for the balanced system remarkably improves the results.

The SOF gain calculation procedure can be summarized as follows. Recall from (16) that $q$ is the number of sensors used:

(1) Start from $q=1$

(2) Solve the output controllability gramian maximization problem for the given $(A, B, \tilde{C})$ and $q$.

(3) Solve the $\mathrm{SOF}$ problem by using the output matrix $C=\operatorname{diag}\{\alpha\} \tilde{C}$ found in Step 2 for the balanced realization of the system.

(4) If $S_{t}$ diverges, increase $q$ by one $(q \leftarrow q+1)$ and go to Step 2.

(5) If $S_{t}$ converges, exit.

\section{Examples}

The proposed method is applied to five different examples. The results are compared with the ones in the referenced papers when possible. The solutions are obtained for the balanced realization of the system. The states corresponding to zero Hankel Singular Values are neglected in the SOF gain calculation. The stability condition in Lemma 2 is satisfied for all the examples considered below.

\section{Example I}

The system matrices and the compared SOF gains are taken from Garcia et al. (2001) and Bara and Boutayeb (2005) that are sub-scripted by $g$ and $b$, respectively 


$$
\begin{aligned}
A_{g} & =\left[\begin{array}{cccc}
0.5 & 0 & 0.2 & 1.0 \\
0 & -0.3 & 0 & 0.1 \\
0.01 & 0.1 & -0.5 & 0 \\
0.1 & 0 & -0.1 & -1.0
\end{array}\right], \\
B_{g} & =\left[\begin{array}{cc}
1 & 0 \\
0 & 1 \\
0 & 0 \\
-1 & 0
\end{array}\right], C_{g}=\left[\begin{array}{llll}
1 & 0 & 0 & 1 \\
1 & 0 & 1 & 1
\end{array}\right], \\
A_{b} & =\left[\begin{array}{lllll}
0.7286 & 0.8840 & 0.1568 & 0.3916 & 0.9398 \\
0.9551 & 0.3472 & 0.4164 & 0.2528 & 0.8328 \\
0.6564 & 0.0595 & 0.0940 & 0.3544 & 0.4700 \\
0.7423 & 0.7184 & 0.4499 & 0.7430 & 0.6299 \\
0.3450 & 0.9582 & 0.8692 & 0.6508 & 0.0582
\end{array}\right], \\
B_{b} & =\left[\begin{array}{cc}
0.5422 & 0.7869 \\
0.4557 & 0.6560 \\
0.8631 & 0 \\
0.8552 & 0.1312 \\
0.4723 & 0.4949
\end{array}\right], \\
C_{b} & =\left[\begin{array}{lllll}
0.0383 & 0.3279 & 0.3137 & 0.4330 & 0.1845 \\
0.2274 & 0.8995 & 0.2517 & 0.8424 & 0.5082
\end{array}\right] .
\end{aligned}
$$

System matrix $A_{g}$ and $A_{b}$ have unstable eigenvalues at -1.0677 and 2.8034 , respectively.

In Garcia et al. (2001), the SOF gain is directly obtained from the system matrices if the system satisfies some strict conditions. The author present three different cases: First one is the case in which both input and outputs are used. In the second and third cases, second input and second output are neglected respectively. The results are compared with these three different cases. The cost function weights are chosen as $Q=5 \times 10^{3} C^{T} C$ and $R=I$. The compared SOF gain and spectral radius are denoted by $K_{g}$ and $\rho_{g}$. The results of our proposed method are denoted by $K$ and $\rho$. The SOF gains and spectral radius $\rho$, of the closed-loop system matrix are given in Table 1. Spectral norm of the closed-loop transfer function $T(z)$ and sensitivity transfer function $S(z)$,

$$
\begin{aligned}
& T(z)=C(z I-(A+B K C))^{-1} B \\
& S(z)=\left(I-K C(z I-A)^{-1} B\right)^{-1}
\end{aligned}
$$

are given in Figures 1 and 2. As shown in these figures, the SOF gain $K$ leads to better robustness measures in terms of high frequency noise rejection and sensitivity to low frequency reference inputs at the cost of decreasing robustness at mid-frequencies. However, $T(z)$ and $S(z)$ can be shaped by adjusting the cost function weight $Q$. Additionally, as the table illustrates, there is a significant improvement in the spectral radius of the closed loop system.

On the other hand, Bara and Boutayeb (2005) formulates the problem as an LMI. Their results are obtained by solving two LMI problems for a particular realization of the system if it satisfies a condition similar to the one in Lemma 2. For Bara's example the results are obtained by choosing $R=I$ and $Q=\gamma C^{T} C$ for several different $\gamma$ values. They are
Table I. Example I: The SOF gains and spectral radius of the closed-loop system matrix for three cases in Garcia et al. (200I).

\begin{tabular}{lllll}
\hline Case & $K_{g}$ & $K$ & $\rho_{g}$ & $\rho$ \\
\hline Ist & {$\left[\begin{array}{ll}-0.717 & -0.283 \\
-1.588 & 1.488\end{array}\right]$} & {$\left[\begin{array}{cc}-0.818 & -0.343 \\
0.509 & -0.937\end{array}\right]$} & 0.793 & 0.476 \\
2nd & {$\left[\begin{array}{ll}-0.325 & -0.650\end{array}\right]$} & {$\left[\begin{array}{ll}-0.632 & -0.551\end{array}\right]$} & 0.590 & 0.491 \\
3rd & {$\left[\begin{array}{l}-0.856 \\
-0.856\end{array}\right]$} & {$\left[\begin{array}{l}-1.184 \\
-0.348\end{array}\right]$} & 0.646 & 0.479 \\
& & {[} & & \\
\hline
\end{tabular}

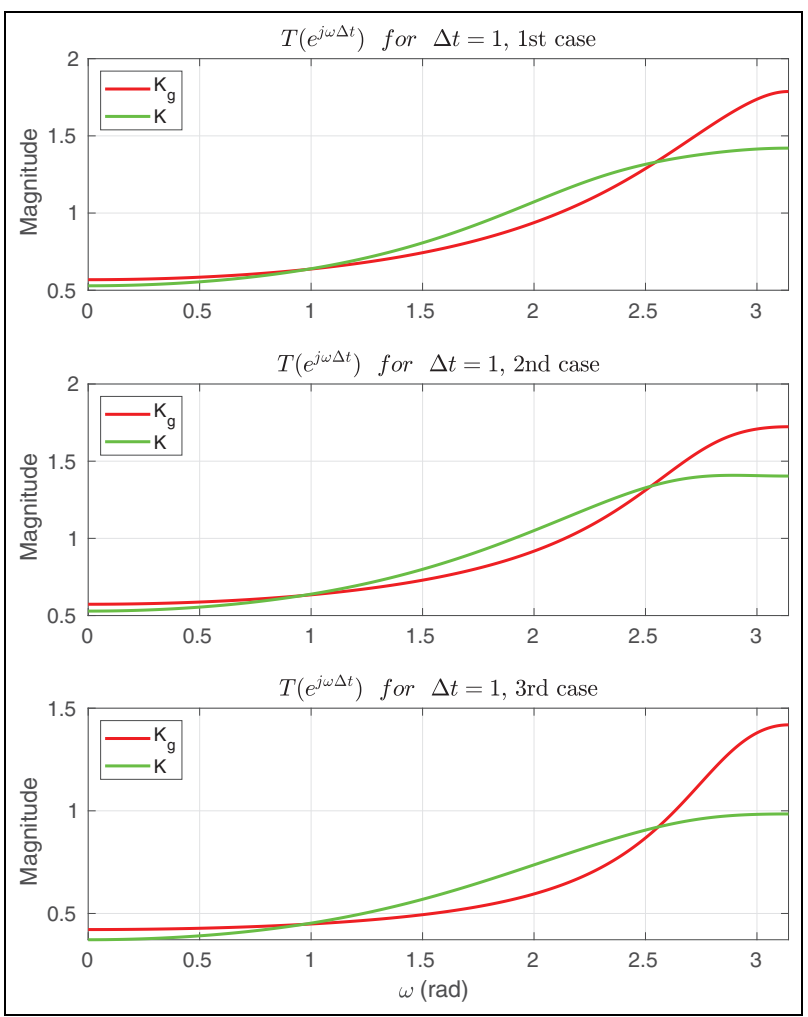

Figure I. Example I: Maximum singular value of the closed-loop transfer function $T(z)$ compared with Garcia et al. (200I).

compared with the result in Bara and Boutayeb (2005), that is given by

$$
K_{b}=\left[\begin{array}{cc}
-0.5045 & -0.9594 \\
0.4777 & -0.4503
\end{array}\right], \rho_{b}=0.5857 .
$$

The SOF gains and spectral radius $\rho$, of the closed-loop system matrix are given in Table 2. Spectral norm of the closedloop transfer function $T(z)$ and sensitivity transfer function $S(z)$, are given in Figures 3 and 4. The spectral radius exceeds $\rho_{b}$ when $\gamma=10$ but there is a big improvement in the sensitivity at low frequencies for all $\gamma$.

\section{Example 2: Aircraft model}

The second example is the continuous time model of the lateral-directional command augmentation system of an F-16 


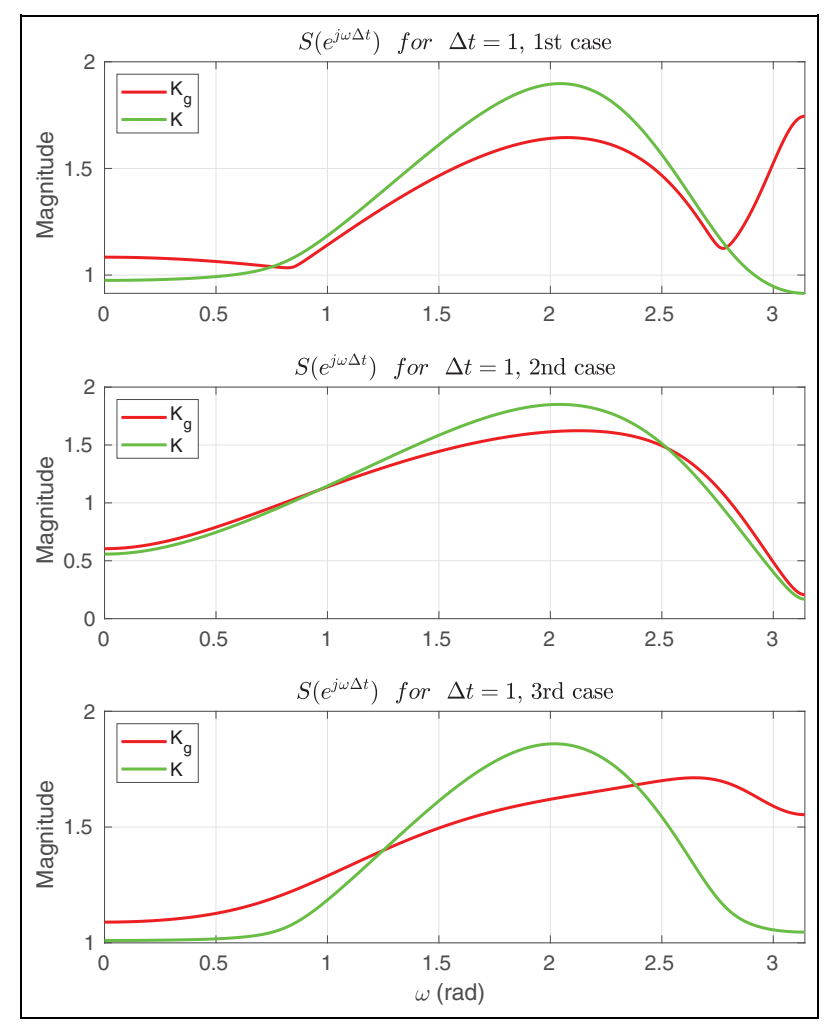

Figure 2. Example I: Maximum singular value of the sensitivity transfer function S(z) compared with Garcia et al. (200I).

aircraft linearized around its nominal conditions (Gadewadikar et al., 2007; Stevens et al., 2016). The continuous time state space realization $\left(A_{c}, B_{c}, C_{c}\right)$ are given by

$$
\begin{aligned}
& A_{c}=\left[\begin{array}{ccccccc}
-0.3220 & 0.0640 & 0.0364 & -0.9917 & 0.003 & 0.0008 & 0 \\
0 & 0 & 1 & 0.0037 & 0 & 0 & 0 \\
-30.6492 & 0 & -3.6784 & 0.6646 & -0.7333 & 0.1315 & 0 \\
8.5396 & 0 & -0.0254 & -0.4764 & -0.0319 & -0.0620 & 0 \\
0 & 0 & 0 & 0 & -20.2 & 0 & 0 \\
0 & 0 & 0 & 0 & 0 & -20.2 & 0 \\
0 & 0 & 0 & 57.2958 & 0 & 0 & -1
\end{array}\right] \\
& B_{c}=\left[\begin{array}{ccccccc}
0 & 0 & 0 & 0 & 20.2 & 0 & 0 \\
0 & 0 & 0 & 0 & 0 & 20.2 & 0
\end{array}\right]^{T}, \\
& C_{c}=\left[\begin{array}{ccccccc}
0 & 0 & 0 & 57.2958 & 0 & 0 & -1 \\
0 & 0 & 57.2958 & 0 & 0 & 0 & 0 \\
57.2958 & 0 & 0 & 0 & 0 & 0 & 0 \\
0 & 57.2958 & 0 & 0 & 0 & 0 & 0
\end{array}\right] \text {, }
\end{aligned}
$$

$x_{c}=\left[\begin{array}{lllllll}\beta & \phi & p & r & \delta_{a} & \delta_{r} & x_{w}\end{array}\right]$,

where $x_{c}$ is the state vector and the states are side-slip angle $\beta$, bank angle $\phi$, roll rate $p$, yaw rate $r$ (see Figure 5). The variables $\delta_{a}$ and $\delta_{r}$ come from the aileron and rudder actuator models. The washout filter state is denoted by $x_{w}$. They constitute a stable system with the given system matrix $A_{c}$. The model is discretized by zero order hold $(\mathrm{ZOH})$ with the sampling period $h=0.01 \mathrm{sec}$. The discrete time model is

$$
\begin{gathered}
x_{t+1}=A x_{t}+B u_{t} \\
y_{t}=\tilde{C} x_{t}
\end{gathered}
$$

where $A=e^{h A_{c}}, B=\left(\int_{0}^{h} e^{A_{c} \tau} d \tau\right) B_{c}$ and $\tilde{C}=C_{c}$. The results are compared with the discrete time version of the method in Gadewadikar et al. (2007). However, the effect of disturbance in their model is neglected.

In the simulations, the cost function weights are chosen as $Q=\gamma C^{T} C$ and $R=I$. The proposed method will be denoted as $\operatorname{Method}_{1}$ and the compared method is $\operatorname{Method}_{0}$. The results found for different values of $\gamma$ are shown in Table 3. For small values of $\gamma<10$, the spectral radius of the closed loop system matrix for both methods are approximately the same. The spectral radius decreases for Method $_{1}$ as $\gamma$ is increased. For the case in which $\gamma=100$, Method $_{0}$ does not converge.

Furthermore, the SOF gain is calculated by using less than four available outputs after solving the output controllability gramian maximization problem for $q=\{1,2,3\}$. The resulting optimal $\alpha$ vectors are given in Table 4. The most significant output is the bank angle followed by the roll rate and yaw rate. Comparison of the SOF gains and spectral radius of the closed-loop system matrices are in Table 5. Method $_{1}$ gives approximately the same $\rho$ as Method $_{0}$ for all cases, but it has a big advantage as the peak sensitivity is significantly smaller.

\section{Example 3: Aircraft model with actuator failure}

In the continuous time model, the rudder actuator is modeled as $A_{r}(s)=20.2 /(20.2+s)$. In this example, the rudder actuator is assumed to have failed and its model is replaced by $\Sigma_{r}(s)=1 /(s+\epsilon)$ to approximate a stuck actuator integrating (we take $\epsilon=-0.001$ to avoid imaginary axis poles in the system). Stabilization by using a minimum number of outputs is investigated for this unstable aircraft model.

The output controllability gramian maximization problem is solved for different $q$ (see Table 6). When a rudder failure occurs, sensing the side-slip angle is now preferred to the roll rate. The proposed method can find a stabilizing gain for all $q$ values. Results are given in Table 7. $\operatorname{Method}_{0}$ fails to find a stabilizing solution for this unstable aircraft model.

\section{Example 4: Simply supported beam}

The example is taken from Hiramoto et al. (2000). First, 10 natural modes are used to approximate the continuous time transfer function of the simply supported flexible beam with length $L_{b}$, Young's modulus $E_{b}$, moment of inertia $I_{b}$, density $\rho_{b}$ and cross-sectional area $S_{b}$. The natural modes of the beam are represented by the resonance frequencies

$$
\omega_{i}=(i \pi)^{2} \sqrt{\frac{E_{b} I_{b}}{\rho_{b} S_{b} L_{b}^{4}}}
$$

and the damping terms $\zeta_{i}$ for $i=1, \cdots, 10$. The continuous time system's state space matrices are constructed from blocks 
Table 2. Example I: The SOF gains and spectral radius of the closed-loop system matrix for different $\gamma$.

\begin{tabular}{lll}
\hline$\gamma$ & $K$ & $\rho$ \\
\hline $\mathrm{I}$ & {$\left[\begin{array}{ll}-0.6803 & -0.7261 \\
-0.0981 & -0.5116\end{array}\right]$} & 0.5643 \\
10 & {$\left[\begin{array}{cc}-0.9078 & -0.7429 \\
0.1071 & -0.5641\end{array}\right]$} & 0.6110 \\
100 & {$\left[\begin{array}{cc}-0.9927 & -0.7380 \\
0.1930 & -0.5872\end{array}\right]$} & 0.6310 \\
\hline
\end{tabular}

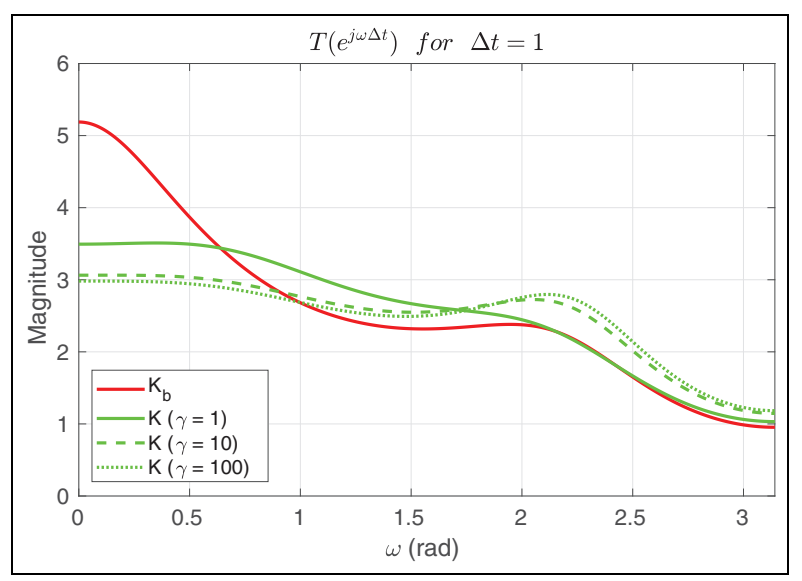

Figure 3. Example I: Maximum singular value of the closed-loop transfer function $T(z)$ compared with Bara and Boutayeb (2005).

$$
\begin{gathered}
A_{i}=\left[\begin{array}{cc}
0 & \omega_{i} \\
-\omega_{i} & -2 \zeta_{i} \omega_{i}
\end{array}\right] \\
B_{i}=\left[\begin{array}{c}
0 \\
H_{i} / \omega_{i}
\end{array}\right], C_{i}=\left[\begin{array}{ll}
0 & \omega_{i} L_{i}
\end{array}\right],
\end{gathered}
$$

where

$$
\begin{gathered}
H_{i}=\left[\begin{array}{llll}
\psi_{i}\left(s_{1}\right) & \psi_{i}\left(s_{2}\right) & \cdots & \psi_{i}\left(s_{m}\right)
\end{array}\right] \\
L_{i}=\left[\begin{array}{llll}
\psi_{i}\left(s_{1}\right) & \psi_{i}\left(s_{2}\right) & \cdots & \psi_{i}\left(s_{r}\right)
\end{array}\right]^{T} \\
\psi_{i}(s)=\sqrt{\frac{2}{L_{b}}} \sin \left(\frac{i \pi s}{L_{b}}\right),
\end{gathered}
$$

and $s$ denotes the position. It is assumed that there are $m$ inputs at positions $s_{i}$ for $i=1, \cdots, m$ and $r$ available output locations at positions $s_{i}$ for $i=1, \cdots, r$ where $s_{i}<s_{i+1} \forall i$. The overall system matrices are

$$
\begin{gathered}
A=\operatorname{blkdiag}\left(A_{i}\right) \text { for } i=1, \cdots, 10, \\
B=\left[\begin{array}{c}
B_{1} \\
B_{2} \\
\vdots \\
B_{10}
\end{array}\right], C=\left[\begin{array}{llll}
C_{1} & C_{2} & \cdots & C_{10}
\end{array}\right] .
\end{gathered}
$$

The parameters of the system are chosen as

\begin{tabular}{|c|c|c|c|c|c|c|}
\hline$\gamma$ & K & & & & & $\rho$ \\
\hline \multirow[t]{2}{*}{ I } & $K_{0}=$ & {$\left[\begin{array}{l}0.2748 \\
0.8208\end{array}\right.$} & $\begin{array}{c}0.6781 \\
-0.1383\end{array}$ & $\begin{array}{c}-1.5333 \\
0.3504\end{array}$ & $\left.\begin{array}{c}0.8157 \\
-0.1500\end{array}\right]$ & $\rho_{0}=0.9885$ \\
\hline & $K_{1}=$ & {$\left[\begin{array}{c}-0.0485 \\
0.3555\end{array}\right.$} & $\begin{array}{c}0.4144 \\
-0.1337\end{array}$ & $\begin{array}{l}-0.3814 \\
-0.0790\end{array}$ & $\begin{array}{c}0.4876 \\
-0.1547\end{array}$ & $\rho_{1}=0.9893$ \\
\hline \multirow[t]{2}{*}{10} & $K_{0}=$ & {$\left[\begin{array}{l}0.7017 \\
2.8201\end{array}\right.$} & $\begin{array}{c}1.5227 \\
-0.3465\end{array}$ & $\begin{array}{l}-2.8858 \\
-0.5327\end{array}$ & $\left.\begin{array}{c}1.7705 \\
-0.3237\end{array}\right]$ & $\rho_{0}=0.9935$ \\
\hline & $K_{1}=$ & {$\left[\begin{array}{c}-8 \times 10^{-4} \\
1.2287\end{array}\right.$} & $\begin{array}{rr}-4 & 0.8738 \\
& -0.4213\end{array}$ & $\begin{array}{ll}3 & -0.2666 \\
3 & -1.1752\end{array}$ & {$\left[\begin{array}{cc}6 & 0.9621 \\
2 & -0.4915\end{array}\right]$} & $\rho_{1}=0.9897$ \\
\hline \multirow[t]{2}{*}{50} & $K_{0}=$ & {$\left[\begin{array}{l}1.2726 \\
5.9145\end{array}\right.$} & $\begin{array}{c}2.0055 \\
-0.5376\end{array}$ & $\begin{array}{l}-4.0221 \\
-3.2253\end{array}$ & $\left.\begin{array}{c}2.3270 \\
-0.4304\end{array}\right]$ & $\rho_{0}=0.9940$ \\
\hline & $K_{1}=$ & {$\left[\begin{array}{l}0.1749 \\
2.5306\end{array}\right.$} & $\begin{array}{c}1.0544 \\
-0.8876\end{array}$ & $\begin{array}{l}-0.5354 \\
-3.6937\end{array}$ & $\begin{array}{c}1.1439 \\
-1.0166\end{array}$ & $\rho_{1}=0.9898$ \\
\hline \multicolumn{7}{|c|}{$100 \operatorname{Method}_{0}$ does not converge. } \\
\hline & $K_{1}=$ & {$\left[\begin{array}{l}0.2896 \\
3.3035\end{array}\right.$} & $\begin{array}{c}1.0569 \\
-1.2179\end{array}$ & $\begin{array}{l}-0.7656 \\
-5.3348\end{array}$ & $\left.\begin{array}{c}1.1433 \\
-1.3803\end{array}\right]$ & $\rho_{1}=0.9898$ \\
\hline \multicolumn{7}{|c|}{500 Method $_{0}$ does not converge. } \\
\hline & $K_{1}=$ & {$\left[\begin{array}{l}0.5684 \\
5.2737\end{array}\right.$} & $\begin{array}{c}0.9565 \\
-2.2629\end{array}$ & $\begin{array}{l}-1.3692 \\
-9.6825\end{array}$ & $\begin{array}{c}1.0320 \\
-2.5113\end{array}$ & $\rho_{\mathrm{I}}=0.9898$ \\
\hline
\end{tabular}
$E_{b}=L_{b}=I_{b}=\rho_{b}=S_{b}=1$ and $\zeta_{i}=0.005 \forall i$. The cost

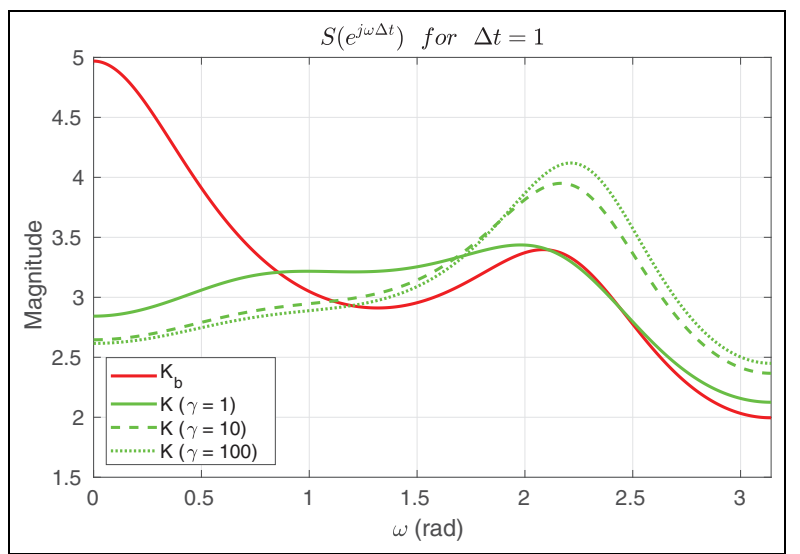

Figure 4. Example I: Maximum singular value of the sensitivity transfer function S(z) compared with Bara and Boutayeb (2005).

Table 3. Example 2: The SOF gains and spectral radius of the closed-loop system matrix for different $\gamma$ values.

Table 4. Example 2: Optimal $\alpha$ vectors in terms of output controllability. Indices of ones in $\alpha$ shows the indices of sensed outputs.

\begin{tabular}{lllll}
\hline$q$ & $\alpha^{T}$ & & & \\
\cline { 2 - 5 } & yaw $\left(r_{w}\right)$ & roll $(p)$ & side-slip $(\beta)$ & $\operatorname{bank}(\phi)$ \\
\hline 1 & {$[0$} & 0 & 0 & $1]$ \\
2 & {$[0$} & 1 & 0 & $1]$ \\
3 & {$[1$} & 1 & 0 & $1]$ \\
\hline
\end{tabular}

function weights are chosen as $Q=I$ and $R=I$. The system is discretized with the sampling period $\Delta \tau=1 \times 10^{-3} \mathrm{sec}$.

It is assumed that four actuators are placed at the positions $0.2,0.4,0.6$ and $0.8 m$. Twenty sensors sites are assigned 


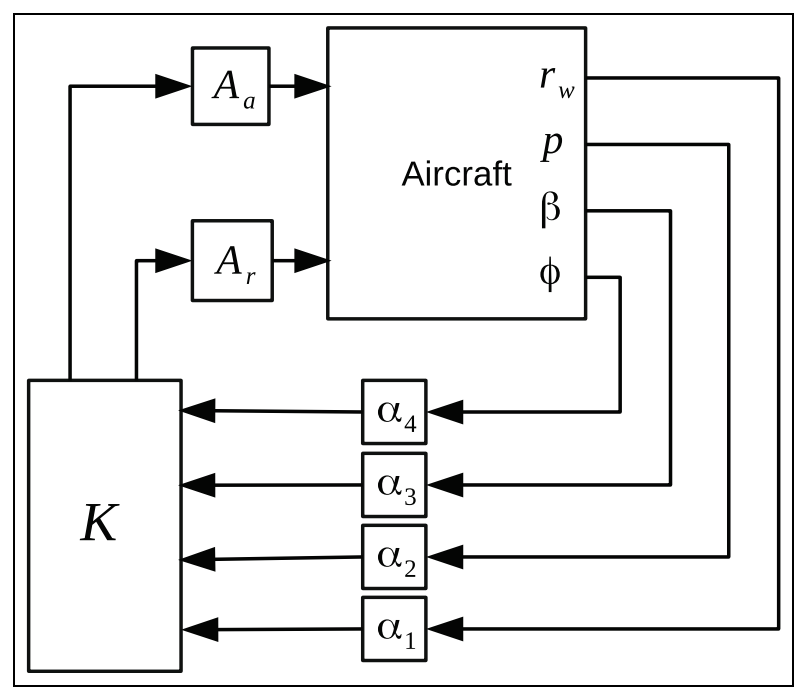

Figure 5. In the aircraft model, $A_{a}$ and $A_{r}$ are aileron and rudder actuators. $\alpha_{i} \in\{0,1\}$ determines that corresponding output is used for feedback.

at equidistant points between 0 and $L_{b}=1 \mathrm{~m}$. The optimal sensor configurations for $q=1, \cdots, 5$ are given in Table 8 . The suppression ratios of the first three natural modes are given in Table 9 where

$$
\begin{gathered}
P(z)=C(z I-A)^{-1} B \\
T(z)=C(z I-(A+B K C))^{-1} B .
\end{gathered}
$$

Table 9 shows that using larger number of sensors leads to more efficient suppression of modes.

Then, an unstable beam is considered by introducing a negative damping to one of the natural modes. In this case, the unstable system can be stabilized by using a few outputs that is obtained from the solution of optimal sensor problem. The results for this case are given in Table 10 .

\section{Example 5: Biological network}

Our last example is a linearized version of the partial differential equation (PDE) that models aggregation of cellular slime molds taken from Edelstein-Keshet (2005). According to the model, slime molds produce cAMP chemical which attracts the slime mold cells and leads to the aggregation of cells. The cAMP concentration decreases with respect to a decay rate. In the model, $s$ and $\tau$ are continuous position and time variables. $a(s, \tau)$ is the density of slime molds and $c(s, \tau)$ is the concentration of cAMP chemical

$$
\begin{gathered}
\frac{\partial a(s, \tau)}{\partial \tau}=\mu \frac{\partial^{2} a(s, \tau)}{\partial s^{2}}-\chi \bar{a} \frac{\partial^{2} c(s, \tau)}{\partial s^{2}} \\
\frac{\partial c(s, \tau)}{\partial \tau}=D \frac{\partial^{2} c(s, \tau)}{\partial s^{2}}+f(s) a(s, \tau)-k(s) c(s, \tau),
\end{gathered}
$$

where $\mu$ determines the cell mobility, $\chi$ is the chemotactic coefficient, $D$ is the diffusion rate of cAMP, $f(s)$ and $k(s)$ are
cAMP generation and decay rates. The PDE is discretized with spatial period $\Delta s$. The discretized version is given by

$$
\begin{aligned}
& \frac{a(i, \tau)}{d \tau}=\mu \frac{a(i+1, \tau)-2 a(i, \tau)+a(i-1, \tau)}{\Delta s^{2}} \\
& -\chi \bar{a} \frac{c(i+1, \tau)-2 c(i, \tau)+c(i-1, \tau)}{\Delta s^{2}} \\
& \frac{c(i, \tau)}{d \tau}=D \frac{c(i+1, \tau)-2 c(i, \tau)+c(i-1, \tau)}{\Delta s^{2}} \\
& +f(i) a(i, \tau)-k(i) c(i, \tau),
\end{aligned}
$$

where $a(i, \tau)=a(i \Delta s, \tau), c(i, \tau)=c(i \Delta s, \tau)$. In the state space form

$$
\begin{gathered}
\frac{d x_{c}}{d \tau}(i)=\left[\begin{array}{cc}
-2 \mu / \Delta s^{2} & 2 \chi \bar{a} / \Delta s^{2} \\
f(i) & -2 D / \Delta s^{2}-k(i)
\end{array}\right] x_{c}(i) \\
+\left[\begin{array}{cc}
\mu / \Delta s^{2} & -\chi \bar{a} / \Delta s^{2} \\
0 & D / \Delta s^{2}
\end{array}\right] x_{c}(i-1) \\
+\left[\begin{array}{cc}
\mu / \Delta s^{2} & -\chi \bar{a} / \Delta s^{2} \\
0 & D / \Delta s^{2}
\end{array}\right] x_{c}(i+1) \\
\dot{x}_{c}(i)=A_{i} x(i)+M_{i, i-1} x_{c}(i-1)+M_{i, i+1} x_{c}(i+1), \\
\text { fori }=1, \cdots, N,
\end{gathered}
$$

where $x_{c}(i)=\left[\begin{array}{ll}a(i, \tau) & c(i, \tau)\end{array}\right]^{T}$. It is assumed that for some $i \in \mathbb{Y}$ the concentration of slime molds can be sensed and for some $i \in \mathbb{U}$, the cAMP concentration can be modified externally. In particular

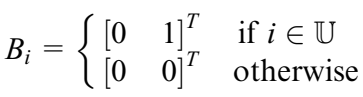

$$
\begin{aligned}
& C_{i}= \begin{cases}{\left[\begin{array}{ll}
1 & 0
\end{array}\right]} & \text { if } i \in \mathbb{Y} \\
{\left[\begin{array}{ll}
0 & 0
\end{array}\right]} & \text { otherwise }\end{cases}
\end{aligned}
$$

State vectors $x(i)$ of the subsystems can be combined in a large scale system with sparse $A_{c}, B_{c}$ and $C_{c}$ matrices. The subsystems are connected to each other to create a ring shaped structure (Figure 7b). Nonzero structure of the system matrix $A_{c}$ can be found in Figure 8 . The symmetry is broken by scaling $M_{1,17}$ by a factor of 0.95 to eliminate poles on the imaginary axis. Continuous time model is discretized by the sampling rate $h=0.01 \mathrm{sec}$. In the simulations, parameters are chosen as

$$
\begin{gathered}
\Delta s=1 \times 10^{-4}, \mu=1 \times 10^{-7}, \chi=4 \times 10^{-4}, \\
\bar{a}=1.6 \times 10^{-3}, D=3 \times 10^{-8}, k(i)=1.5, f(i)=0.3, \\
R=I, Q=I .
\end{gathered}
$$

In the ring, there is an anomalous subsystem that generates cAMP with a higher rate and destabilizes the network. The generation rate is $f(i)=0.6$ for this anomalous subsystem. In Figure 8 , destabilizing subsystem $\left(A_{9}\right)$ is shown by the box with dashed pattern. There are two gray boxes, $A_{1}$ is connected to the first input and $A_{17}$ connected to the second input. The boxes with gray edges are the subsystems from which slime mold density is sensed.

The system matrix $A$ has 3 unstable eigenvalues at $1.00145,1.00007,1.00006$. The proposed SOF calculation 
Table 5. Example 2: Comparison of the SOF gains, the sensitivity transfer function peak $\|S(z)\|_{\infty}$ and the spectral radius of the closed-loop system matrix $\rho$ found by Method $d_{0}$ and Method, for $\gamma=I$ and different sensors configurations given in Table 4 .

\begin{tabular}{|c|c|c|c|c|c|c|c|c|c|}
\hline$q$ & $K_{0}$ & & $\rho_{0}$ & $\left\|S_{0}(z)\right\|_{\infty}$ & $K_{\mathrm{I}}$ & & & $\rho_{1}$ & $\left\|S_{I}(z)\right\|_{\infty}$ \\
\hline 1 & {$\left[\begin{array}{c}0.8172 \\
-0.4283\end{array}\right]$} & & 0.9950 & 1.72 & {$\left[\begin{array}{l}0.31178 \\
-0.2233\end{array}\right]$} & & & 0.9919 & 1.45 \\
\hline 2 & $\begin{array}{cc}0.6794 & 0.8135 \\
-0.1390 & -0.1897\end{array}$ & & 0.9953 & 2.70 & $\begin{array}{l}0.4270 \\
-0.108 I\end{array}$ & $\left.\begin{array}{c}0.5025 \\
-0.1431\end{array}\right]$ & & 0.9953 & 1.98 \\
\hline 3 & $\begin{array}{cc}0.2059 & 0.6786 \\
0.7516 & -0.1445\end{array}$ & $\left.\begin{array}{c}0.8121 \\
-0.2021\end{array}\right]$ & 0.9895 & 3.44 & $\begin{array}{c}-0.0641 \\
0.3644\end{array}$ & $\begin{array}{l}0.4176 \\
-0.1368\end{array}$ & $\left.\begin{array}{c}0.4850 \\
-0.1623\end{array}\right]$ & 0.9894 & 2.48 \\
\hline
\end{tabular}

Table 6. Example 3: Optimal $\alpha$ vectors in terms of the output controllability for the unstable aircraft model. Ones in $\alpha$ show the indices of sensed outputs.

\begin{tabular}{lllll}
\hline$q$ & $\alpha^{T}$ & & & \\
\cline { 2 - 5 } & yaw $\left(r_{w}\right)$ & roll $(p)$ & side-slip $(\beta)$ & bank $(\phi)$ \\
\hline 1 & {$[0$} & 0 & 0 & $1]$ \\
2 & {$[0$} & 0 & 1 & $1]$ \\
3 & {$[0$} & 1 & 1 & $1]$ \\
\hline
\end{tabular}

Table 7. Example 3: The SOF gains and spectral radius of the closedloop system matrix found when $Q=I_{n}$ for the unstable aircraft model.

\begin{tabular}{lll}
\hline$q$ & $K_{1}$ & $\rho_{1}$ \\
\hline 1 & {$\left[\begin{array}{l}0.1510 \\
0.0123\end{array}\right]$} & 0.9999 \\
2 & {$\left[\begin{array}{lll}-2.3592 & 0.1921 \\
-0.4587 & 0.0195\end{array}\right]$} & 0.9990 \\
3 & {$\left[\begin{array}{lll}0.2082 & -6.2837 & 0.2926 \\
0.0246 & -1.1207 & 0.0347\end{array}\right]$} & 0.9984 \\
\hline
\end{tabular}

Table 8. Example 4: Optimal sensor locations for the simply supported beam.

\begin{tabular}{lllll}
\hline$q$ & Positions $(m)$ & \\
\hline 1 & {$[0.60]$} & & & \\
2 & {$\left[\begin{array}{lllll}0.55 & 0.60\end{array}\right]$} & & \\
3 & {$\left[\begin{array}{lllll}0.45 & 0.50 & 0.55\end{array}\right]$} & \\
4 & {$\left[\begin{array}{lllll}0.45 & 0.50 & 0.55 & 0.60\end{array}\right]$} \\
5 & {$\left[\begin{array}{lllll}0.45 & 0.50 & 0.55 & 0.60 & 0.65\end{array}\right]$} \\
\hline
\end{tabular}

method converges when $q=6$ and the corresponding optimal sensor locations are given by indices $\mathbb{Y}=\{1,3,4,5,9,17\}$. The sensors are not distributed symmetrically due to the broken symmetry of the network. Finally, the stabilizing SOF gain is

$K=\left[\begin{array}{cccccc}-0.5032 & -4.1974 & 6.3807 & -9.1076 & -5.2413 & 0.9617 \\ 1.2853 & 9.6815 & -12.8261 & 21.6236 & 15.9835 & -1.6436\end{array}\right]$
Table 9. Example 4: The ratios $T(z) / P(z)$ of the open-loop $P(z)$ and closed-loop transfer functions $T(z)$ at the first three natural frequencies.

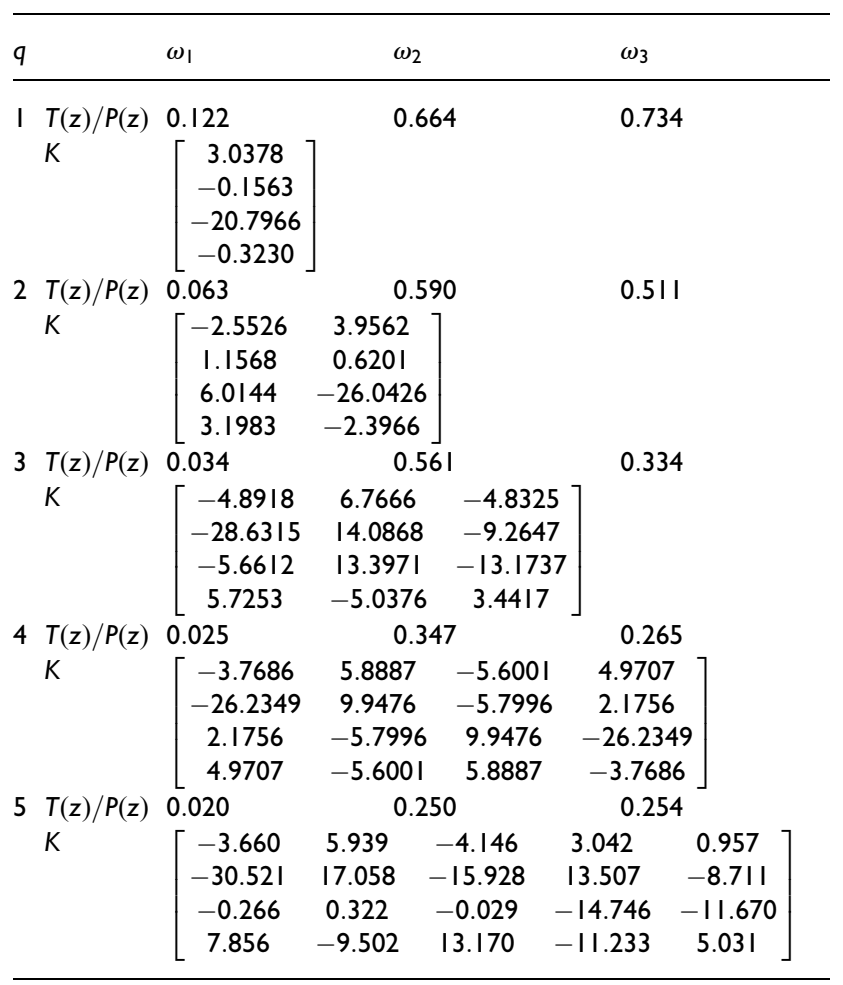

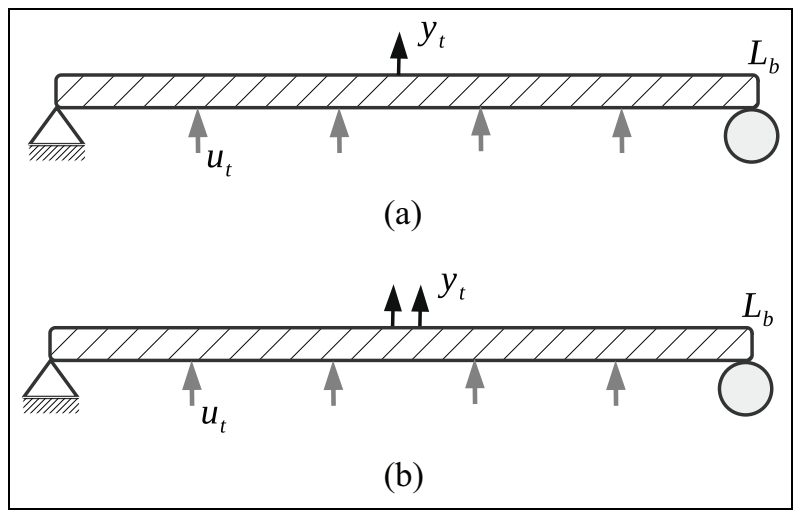

Figure 6. Example 4: Illustration of the sensor and actuator locations for the unstable flexible beam example: When the unstable mode is $\zeta_{1}=-0.005$ in (6) and $\zeta_{10}=-0.005$ in (7).

Gray arrows show the input locations and black arrows are the outputs. 
Table 10. Example 4: The optimal sensor positions and spectral radius of the closed-loop system matrix for the unstable beam for two different unstability conditions where $\rho(A)$ is spectral radius of the unstable openloop system matrix (see Figure 6 for an illustration of the actuator/ sensor locations).

\begin{tabular}{llll}
\hline Unstable mode & Positions $(m)$ & $K$ & $\rho$ \\
\hline$\zeta_{1}=-0.005$ & {$[0.45]$} & {$\left[\begin{array}{c}-0.3260 \\
-20.8119 \\
-0.1653 \\
3.0313\end{array}\right]$} & 0.9988 \\
$\rho(A)=1.00004$ & & {$\left[\begin{array}{cc}-3.2073 & 8.0214 \\
-28.363 & 12.664 \\
-1.0227 & 1.3668 \\
3.9430 & -5.5116\end{array}\right]$} & \\
$\zeta_{10}=-0.005$ & {$[0.45$} & $0.50]$ & \\
$\rho(A)=1.0071$ & & &
\end{tabular}

which results to a spectral radius of $\rho=0.9994$.

\section{Discussion}

The proposed algorithm yields promising results but the convergence of the approximate solution of LQR problem is not easily tractable. In Bertsekas (1995), a proof of convergence is given when $(A, B)$ is controllable and $(A, Q)$ is observable for $Q=C^{T} C$. Controllability condition is relaxed and replaced by stabilizability in Lancaster and Rodman (1995). Nevertheless, the method described in this study considers a sub-optimal state feedback gain in the form of $\hat{F}=K C$ where $K=F C^{\dagger}$. It can also be represented by

$$
\hat{F}=F C^{\dagger} C=F \Pi_{c},
$$

where $\Pi_{c}$ is the orthogonal projection matrix on $\operatorname{range}\left(C^{T}\right)$.

Remark 2. Equation (22) can be equivalently written as

$$
\begin{aligned}
S & =Q+A^{T}\left(S-S B \bar{R}^{-1} B^{T} S\right) A \\
& +\prod_{\bar{c}} A^{T} S B \bar{R}^{-1} B^{T} S A \Pi_{\bar{c}},
\end{aligned}
$$

where $\bar{R}=R+B^{T} S B$ and $\Pi_{\bar{c}}=I_{n}-\Pi_{c}$ is the orthogonal projection on $\operatorname{null}(C)$. Hence, the proposed SOF calculation method satisfies the following DARE

$$
S=\tilde{Q}+A^{T}\left(S-S B \bar{R}^{-1} B^{T} S\right) A,
$$

where $\tilde{Q}=Q+\Pi_{\bar{c}} A^{T} S B \bar{R}^{-1} B^{T} S A \Pi_{\bar{c}} \geqslant Q$.

Assume that the second term in $\tilde{Q}$ is already known, the total cost $V$ of the LQR problem with the cost function

$$
V=x_{t_{f}}^{T} \tilde{Q} x_{t_{f}}+\sum_{t=0}^{t_{f}-1} x_{t}^{T} \tilde{Q} x_{t}+u_{t}^{T} R u_{t}
$$

is given by

$$
\begin{aligned}
V & =x_{t_{f}}^{T} \tilde{Q} x_{t_{f}}+\sum_{t=0}^{t_{f}-1} x_{t}^{T}\left(\tilde{Q}+F^{T} R F\right) x_{t} \\
& =x_{t_{f}}^{T} \tilde{Q} x_{t_{f}}+\sum_{t=0}^{t_{f}-1} x_{t}^{T}\left(S-(A+B F)^{T} S(A+B F)\right) x_{t} \\
& =x_{t_{f}}^{T} \tilde{Q} x_{t_{f}}+\sum_{t=0}^{t_{f}-1} x_{t}^{T} S x_{t}-x_{t+1}^{T} S x_{t+1} \\
& =x_{0}^{T} S x_{0}+x_{t_{f}}^{T}(\tilde{Q}-S) x_{t_{f}},
\end{aligned}
$$

when $u_{t}=-\bar{R}^{-1} B^{T} S A x_{t}=F x_{t}$. Our method proposes a suboptimal state feedback $\hat{u}_{t}=F \Pi_{c} x_{t}=\hat{F} x_{t}$. Similarly, the total cost $\hat{V}$ for the input $\hat{u}_{t}$ can be written as

$$
\begin{aligned}
\hat{V} & =x_{t_{f}}^{T} Q x_{t_{f}}+\sum_{t=0}^{t_{f}-1} x_{t}^{T} Q x_{t}+\hat{u}_{t}^{T} R \hat{u}_{t} \\
& =x_{0}^{T} S x_{0}+x_{t_{f}}^{T}(Q-S) x_{t_{f}} .
\end{aligned}
$$

Since the closed loop system is stable for both cases, $V \approx \hat{V}$ for an arbitrarily large $t_{f}$. Eventually, it can be said that the proposed algorithm leads to a similar quadratic cost as the LQR problem with a larger weight $(\tilde{Q})$ on the system's states.

Additionally, efficiency of the proposed method for sensor placement can be demonstrated by solving the SOF problem for non-optimal sensor sets. It is observed that the proposed SOF calculation converges slower to a worse minimum when other possible $\alpha$ configurations are used. The results for non-optimal sensor placement combinations for the unstable aircraft model are given in Table 11.

\section{Conclusion}

In this paper, the SOF problem is investigated along with the optimal selection of the system outputs. First, the optimal sensor placement problem by the output controllability gramian maximization is described and its relation with the mini-

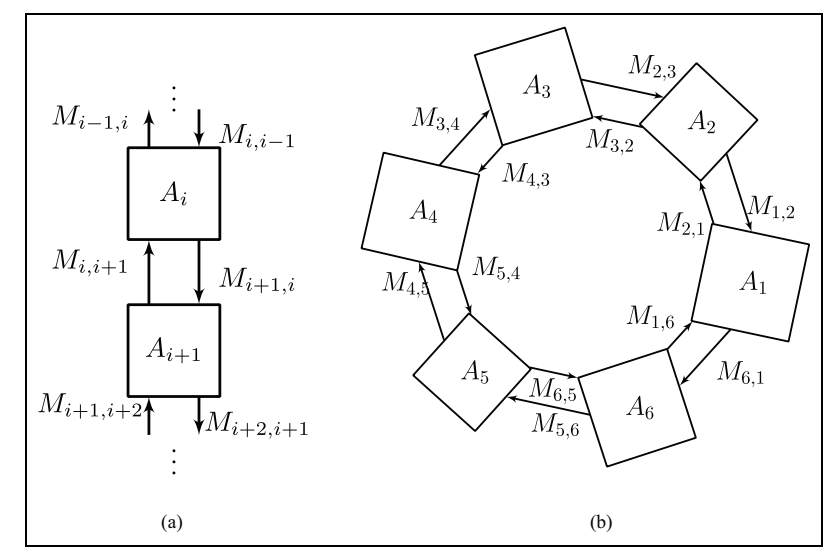

Figure 7. Example 5: (7a) Interconnection of the subsystems at discrete positions. (7b) The circular interconnection of systems. 


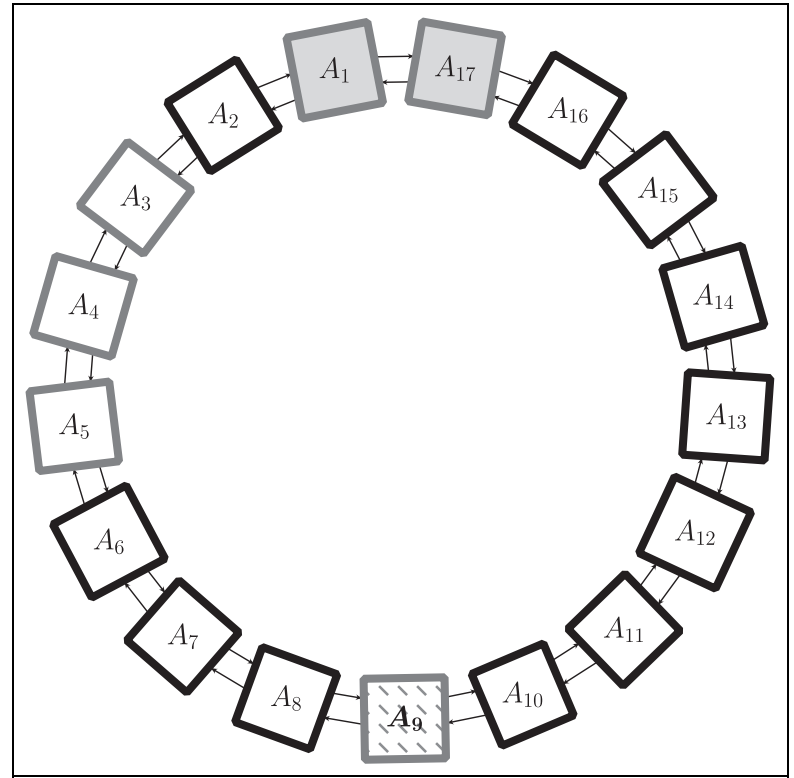

Figure 8. Example 5: The ring network for $N=17$ is shown. The box with dashed pattern $\left(A_{9}\right)$ represents the anomalous subsystem, the gray boxes $\left(A_{1}, A_{17}\right)$ are subsystems where the inputs are applied. The sensors are placed on the boxes with gray borders $\left(A_{1}, A_{3}, A_{4}, A_{5}, A_{9}, A_{17}\right)$.

Table II. Example 3: The resulting spectral radius of the closed-loop system matrices for non-optimal $\alpha$ configurations.

\begin{tabular}{|c|c|c|c|c|}
\hline \multicolumn{4}{|l|}{$\alpha^{T}$} & \multirow{2}{*}{$\begin{array}{l}\rho \\
S_{t} \text { does not converge. }\end{array}$} \\
\hline$[1$ & 0 & 0 & 0] & \\
\hline 0 & 10 & 0 & 0] & $A \Pi_{\bar{c}}$ is unstable and $S_{t}$ does not converge. \\
\hline & 0 & I & 0] & 0.9999 \\
\hline$[1$ & 10 & 0 & 0] & $A \Pi_{\bar{c}}$ is unstable and $S_{t}$ does not converge. \\
\hline$[1$ & 0 & I & 0] & 0.9999 \\
\hline$[1$ & 0 & 0 & $1]$ & 0.9999 \\
\hline$[0$ & 1 & I & 0] & 0.9999 \\
\hline 0 & 10 & 0 & I] & 0.9999 \\
\hline$[1$ & 0 & I & I] & 0.9992 \\
\hline$[1$ & 10 & 0 & I] & 0.9999 \\
\hline
\end{tabular}

mization of the required input energy is shown. Since the system is not necessarily stable, a procedure for calculating the generalized gramians of unstable discrete time LTI systems is developed. Also, it is shown by the examples that norm of the output controllability gramian can be used as a reliable metric to determine optimal sensor locations for the SOF stabilization.

After a full system realization $(A, B, C)$ is obtained by structuring the output matrix $C$, the next problem addressed is to calculate a stabilizing SOF gain. The SOF gain is calculated as a projected solution of the LQR problem by approximate dynamic programming. Efficiency of the sensor placement method and the the SOF calculation method are compared with the examples from the literature in terms of spectral radius and robustness measures. A necessary condition for the existence of such a SOF gain is introduced. It is pointed out that the proposed SOF stabilization method leads to a quadratic cost similar to an LQR problem with a larger cost function weight on the state vector.

The proposed solution for the SOF problem lacks the convergence guarantee similar to the counterparts in the literature. A promising approach would be to use the balanced form of the system. Nevertheless, the convergence characteristics and the optimal selection of the state space realization are still open problems to be studied in the future.

\section{Acknowledgements}

The authors would like to thank the anonymous reviewers for their suggestions. The authors would also like to thank Serdar Yüksel for enlightening discussions on dynamic programming and decentralized control. The first author acknowledges TÜBİTAK for PhD scholarship.

\section{Declaration of conflicting interests}

The author(s) declared no potential conflicts of interest with respect to the research, authorship, and/or publication of this article.

\section{Funding}

The author(s) received no financial support for the research, authorship, and/or publication of this article.

\section{ORCID iD}

Okan Demir (iD https://orcid.org/0000-0003-4380-1425

\section{References}

Bara GI and Boutayeb M (2005) Static output feedback stabilization with $H_{\infty}$ performance for linear discrete-time systems. IEEE Transactions on Automatic Control 50(2): 250-254.

Belabbas MA (2016) Geometric methods for optimal sensor design. Proceedings of the Royal Society A: Mathematical, Physical and Engineering Sciences 472(2185): 20150312.

Belabbas MA and Chen X (2018) Sensor placement for optimal estimation of vector-valued diffusion processes. Systems \& Control Letters 121: 24-30.

Bender D (1987) Lyapunov-like equations and reachability/observability gramians for descriptor systems. IEEE Transaction on Automatic Control 32(4): 343-348.

Bertsekas DP (1995) Dynamic programming and optimal control. Vol. 1. Athena scientific optimization and computation series. Belmont, MA: Athena Scientific.

Casadei G, Canudas-de-Wit C and Zampieri S (2018) Controllability of large-scale networks: An output controllability approach. In: 57th IEEE Conference on Decision and Control, Miami, FL, USA, 17-19 December 2018, pp. 5886-5891. IEEE.

Çela A, Niculescu SI, Natowicz R and Reama A (2018) Controllability and observability gramians as information metrics for optimal design of networked control systems. Mechanical Engineering 140(12): S8-S15.

Chen X, Belabbas MA and Ba T (2019) Controlling and stabilizing a rigid formation using a few agents. SIAM Journal of Control Optimization 57(1): 104-128. 
Crusius CAR and Trofino A (1999) Sufficient LMI conditions for output feedback control problems. IEEE Transactions on Automatic Control 44(5): 1053-1057.

Edelstein-Keshet L (2005) Mathematical Models in Biology. Vancouver, Canada: SIAM.

Fu M (2004) Pole placement via static output feedback is $N P$-hard. IEEE Transactions on Automatic Control 49(5): 855-857.

Gadewadikar J and Lewis FL (2006) Aircraft flight controller tracking design using $H_{\infty}$ static output-feedback. Transactions of the Institute of Measurement and Control 28(5): 429-440.

Gadewadikar J, Lewis FL, Xie L, et al. (2007) Parameterization of all stabilizing $H_{\infty}$ static state-feedback gains: Application to outputfeedback design. Automatica 43(9): 1597-1604.

Gamrath G, Anderson D, Bestuzheva K, et al. (2020) The SCIP Optimization Suite 7.0. Technical report, Optimization Online.

Garcia G, Pradin B and Zeng F (2001) Stabilization of discrete time linear systems by static output feedback. IEEE Transactions on Automatic Control 46(12): 1954-1958.

Garvie MR (2007) Finite-difference schemes for reaction-diffusion equations modeling predator-prey interactions in MATLAB. Bulletin of Mathematical Biology 69(3): 931-956.

$\mathrm{Gu}$ G (1990) On the existence of linear optimal control with output feedback. SIAM Journal on Control and Optimization 28(3): 711-719.

Halevi Y and Wagner-Nachshoni C (2006) Transfer function modeling of multi-link flexible structures. Journal of Sound and Vibration 296(1): 73-90.

Hiramoto K, Doki H and Obinata G (2000) Optimal sensor/actuator placement for active vibration control using explicit solution of algebraic riccati equation. Journal of Sound and Vibration 229(5): 1057-1075.

Klickstein I, Shirin A and Sorrentino F (2017) Energy scaling of targeted optimal control of complex networks. Nature Communications 8.

Lagoudakis MG and Parr R (2003) Least-squares policy iteration. Journal of Machine Learning Research 4(Dec): 1107-1149.

Lancaster P and Rodman L (1995) Algebraic Riccati Equations. New York: Oxford University Press.

Lewis FL, Vrabie D and Syrmos VL (1995) Optimal Control. 2nd edition. New York: Wiley.

Liu YY, Slotine JJ and Barabási AL (2011) Controllability of complex networks. Nature 473(7346): 167-173.

Marx B, Koenig D and Georges D (2002) Optimal sensor/actuator location for descriptor systems using Lyapunov-like equations. In: Proceedings of the 41st IEEE Conference on Decision and Control, Las Vegas, NV, USA, 10-13 December 2002, volume 4. pp. 4541-4542. IEEE.

Mercado A and Liu KR (2001) NP-hardness of the stable matrix in unit interval family problem in discrete time. Systems \& Control Letters 42(4): 261-265.

Müller FJ and Schuppert A (2011) Few inputs can reprogram biological networks. Nature 478(7369): E4-E4.

Nemirovskii A (1993) Several NP-hard problems arising in robust stability analysis. Mathematics of Control, Signals and Systems 6(2): 99-105.

Palacios-Quiñonero F, Rubió-Massegú J, Rossell J and Karimi H (2014) Feasibility issues in static output-feedback controller design with application to structural vibration control. Journal of the Franklin Institute 351(1): 139-155.

Palacios-Quiñonero F, Rubió-Massegú J, Rossell JM and Karimi HR (2012) Discrete-time static output-feedback semi-decentralized $H_{\infty}$ controller design: An application to structural vibration control. In: 2012 American Control Conference, Montreal, CA, 27-29 June 2012, pp. 6126-6131. IEEE.
Polyak BT and Shcherbakov PS (2005) Hard problems in linear control theory: Possible approaches to solution. Automation and Remote Control 66(5): 681-718.

Rosinová D, Vesel V and Kučera V (2003) A necessary and sufficient condition for static output feedback stabilizability of linear discrete-time systems. Kybernetika 39(4): 447-459.

Rugh WJ (1996) Linear System Theory. Vol. 2. Upper Saddle River, NJ: Prentice Hall.

Sadabadi MS and Peaucelle D (2016) From static output feedback to structured robust static output feedback: A survey. Annual Reviews in Control 42(2016): 11-26.

Shaker HR and Tahavori M (2013) Optimal sensor and actuator location for unstable systems. Journal of Vibration and Control 19(12): 1915-1920.

Smith M and Sondergeld K (1986) On the order of stable compensators. Automatica 22(1): $127-129$.

Stevens BL, Lewis FL and Johnson EN (2016) Aircraft Control and Simulation: Dynamics, Controls Design, and Autonomous Systems. New Jersey: John Wiley \& Sons.

Summers TH, Cortesi FL and Lygeros J (2016) On submodularity and controllability in complex dynamical networks. IEEE Transactions on Control of Network Systems 3(1): 91-101.

Summers TH and Lygeros J (2014) Optimal sensor and actuator placement in complex dynamical networks. IFAC Proceedings Volumes 47(3): 3784-3789.

Toker O and Özbay H (1995) On the NP-hardness of solving bilinear matrix inequalities and simultaneous stabilization with static output feedback. In: Proceedings of 1995 American Control Conference, Seattle, WA, USA, 21-23 June 1995, volume 4, pp. 25252526. IEEE.

Trofino-Neto A and Kučera V (1993) Stabilization via static output feedback. IEEE Transactions on Automatic Control 38(5): 764-765.

Turing AM (1990) The chemical basis of morphogenesis. Bulletin of Mathematical Biology 52(1): 153-197.

van de Wal M and de Jager B (2001) A review of methods for input/ output selection. Automatica 37(4): 487-510.

Vicsek T and Zafeiris A (2012) Collective motion. Physics Reports 517(3): 71-140.

Yücesoy V and Özbay H (2019) On the real, rational, bounded, unit interpolation problem in and its applications to strong stabilization. Transactions of the Institute of Measurement and Control 41(2): 476-483.

Zhang M and Morris K (2018) Sensor choice for minimum error variance estimation. IEEE Transactions on Automatic Control 63(2): 315-330.

Zhou K, Doyle J and Glover K (1996) Robust and Optimal Control. Volume 40. New Jersey: Prentice Hall.

Zhou K, Salomon G and Wu E (1999) Balanced realization and model reduction for unstable systems. International Journal of Robust and Nonlinear Control 9(3): 183-198.

\section{Appendix}

\section{Proof of Lemma I}

Proof: Proof is based on the solution of optimal state feedback problem with fixed terminal condition in Lewis et al. (1995: Chapter 4). Start by defining quadratic cost

$$
\min _{u_{t}} J=\frac{1}{2} x_{t_{f}}^{T} S_{t_{f}} x_{t_{f}}+\frac{1}{2} \sum_{t=0}^{t_{f}-1} u_{t}^{T} u_{t}
$$


with terminal condition $y_{f}=y_{t_{f}}=C x_{t_{f}}$, which can be equivalently written with Lagrange multipliers $\lambda_{t}$ and $\nu$

$$
\begin{aligned}
J & =\frac{1}{2} x_{t_{f}}^{T} S_{t_{f}} x_{t_{f}}+\left(y_{f}-C x_{t_{f}}\right)^{T} \nu \\
& +\sum_{t=0}^{t_{f}} \frac{1}{2} u_{t}^{T} u_{t}+\lambda_{t+1}\left(A x_{t}+B u_{t}-x_{t+1}\right) .
\end{aligned}
$$

The Hamiltonian is given by

$$
H_{t}=\frac{1}{2} u_{t}^{T} u_{t}+\lambda_{t+1}^{T}\left(A x_{t}+B u_{t}\right)
$$

where the optimal solution satisfies

$$
\begin{gathered}
x_{t+1}=\frac{\partial H_{t}}{\partial \lambda_{t+1}}=A x_{t}+B u_{t} \\
\lambda_{t}=\frac{\partial H_{t}}{\partial x_{t}}=A^{T} \lambda_{t+1},
\end{gathered}
$$

with $u_{t}$ is given by

$$
u_{t}=-B^{T} \lambda_{t+1}
$$

By considering the fixed terminal condition $y_{t_{f}}=y_{f}$, guess a solution given by

$$
\lambda_{t}=S_{t} x_{t}+V_{t} \nu
$$

where $S_{t}$ and $V_{t}$ are (Lewis et al., 1995)

$$
\begin{gathered}
S_{t}=A^{T} S_{t+1}\left(A+B F_{t}\right), \text { for a given } S_{t_{f}}, \\
V_{t}=\left(A+B F_{t}\right)^{T} V_{t+1}, V_{t_{f}}=C^{T},
\end{gathered}
$$

where $F_{t}=-\left(I+B^{T} S_{t+1} B\right) B^{T} S_{t+1} A$. If $V_{t}$ is iterated backwards, we obtain

$$
V_{t}=\Phi\left(t_{f}, t\right)^{T} C^{T}
$$

where

$$
\Phi(t, \tau)=\left(A+B F_{t-1}\right)\left(A+B F_{t-2}\right) \cdots\left(A+B F_{\tau}\right),
$$$$
\text { for } \tau<t \text {. }
$$

Optimal $u_{t}(24)$ can be written as

$$
\begin{aligned}
u_{t} & =-B^{T}\left(S_{t+1} x_{t+1}+V_{t+1} \nu\right) \\
& =-B^{T}\left(S_{t+1}\left(A x_{t}+B u_{t}\right)+V_{t+1} \nu\right) \\
& =-\left(I+B^{T} S_{t+1} B\right)^{-1} B^{T} S_{t+1} A \\
& -\left(I+B^{T} S_{t+1} B\right)^{-1} B^{T} V_{t+1} \nu \\
& =F_{t} x_{t}+r_{t}=w_{t}+r_{t} .
\end{aligned}
$$

The boundary condition $\nu$ can be found from the system's output response at time $t_{f}$ for the input $u_{t}=w_{t}+r_{t}$.

$$
\begin{aligned}
& x_{t_{f}}= \Phi\left(t_{f}, 0\right) x_{0}+\sum_{\tau=0}^{t_{f}-1} \Phi\left(t_{f}, \tau+1\right) B r_{\tau} \\
&=\Phi\left(t_{f}, 0\right) x_{0}-\sum_{\tau=0}^{t_{f}-1} \Phi\left(t_{f}, \tau+1\right) \\
& \times B\left(I+B^{T} S_{\tau+1} B\right)^{-1} B^{T} V_{t+1} \nu \\
&=\Phi\left(t_{f}, 0\right) x_{0}-\sum_{\tau=0}^{t_{f}-1} \Phi\left(t_{f}, \tau+1\right) \\
& \times B\left(I+B^{T} S_{\tau+1} B\right)^{-1} B^{T} \Phi\left(t_{f}, \tau+1\right)^{T} C^{T} \nu \\
& y_{t_{f}}=C \Phi\left(t_{f}, 0\right) x_{0}-Y_{c}\left(t_{f}\right) \nu,
\end{aligned}
$$

where

$$
\begin{aligned}
Y_{c}\left(t_{f}\right) & =C \sum_{\tau=0}^{t_{f}-1} \Phi\left(t_{f}, \tau+1\right) \\
& \times B\left(I+B^{T} S_{\tau+1} B\right)^{-1} B^{T} \Phi\left(t_{f}, \tau+1\right)^{T} C^{T} .
\end{aligned}
$$

That leads to

$$
\nu=Y_{c}\left(t_{f}\right)^{-1}\left(C \Phi\left(t_{f}, 0\right) x_{0}-y_{t_{f}}\right) .
$$

For simplicity, let us choose $S_{t_{f}}=S$ of (6) and $x_{0}=0$. Then

$$
\begin{gathered}
S_{t}=S \forall t \leqslant t_{f} \\
F_{t}=F=-\left(I+B^{T} S B\right)^{-1} B^{T} S A \\
\Phi(t, \tau+1)=(A+B F)^{t-\tau-1}=A_{c l}^{t-\tau-1} \\
u_{t}=F x_{t}+r_{t},
\end{gathered}
$$

where

$$
r_{t}=-\left(I+B^{T} S B\right)^{-1} B^{T}\left(A_{c l}^{T}\right)^{t_{f}-\tau-1} C^{T} Y_{c}\left(t_{f}\right)^{-1} y_{f}
$$

Substitute $u_{t}$ into the cost function (23)

$$
\begin{gathered}
J=\frac{1}{2} x_{t_{f}}^{T} S_{t_{f}} x_{t_{f}}+\frac{1}{2} \sum_{t=0}^{t_{f}-1} J_{t} \\
J_{t}=w_{t}^{T} w_{t}+2 r_{t}^{T} w_{t}+r_{t}^{T} r_{t} .
\end{gathered}
$$

From (24)

$$
w_{t}=-B^{T}\left(S x_{t+1}+V_{t+1} \nu\right)-r_{t}
$$

Then

$$
\begin{aligned}
r_{t}^{T} w_{t} & =-r_{t}^{T} B^{T} S x_{t+1}-r_{t}^{T} B^{T} V_{t+1} v-r_{t}^{T} r_{t} \\
& =-r_{t}^{T} B^{T} S x_{t+1}+r_{t}^{T}\left(I+B^{T} S B\right) r_{t}-r_{t}^{T} r_{t},
\end{aligned}
$$

is obtained. By adding and subtracting $x_{t+1}^{T} S x_{t+1}, J_{t}$ can be simplified 


$$
\begin{aligned}
J_{t} & =w_{t}^{T} w_{t}-2 r_{t}^{T} B^{T} S x_{t+1}+2 r_{t}^{T}\left(I+B^{T} S B\right) r_{t} \\
& -2 r_{t}^{T} r_{t}+r_{t}^{T} r_{t}-x_{t+1}^{T} S x_{t+1}+x_{t+1}^{T} S x_{t+1} \\
& =x_{t}^{T} F^{T} F x_{t}-x_{t+1}^{T} S x_{t+1}+x_{t+1}^{T} S x_{t+1} \\
& -2 r_{t}^{T} B^{T} S x_{t+1}+r_{t}^{T} B^{T} S B r_{t}+r_{t}^{T}\left(I+B^{T} S B\right) r_{t} \\
& =x_{t}^{T} F^{T} F x_{t}+\left(x_{t+1}-B r_{t}\right)^{T} S\left(x_{t+1}-B r_{t}\right) \\
& -x_{t+1}^{T} S x_{t+1}+r_{t}^{T}\left(I+B^{T} S B\right) r_{t} \\
& =x_{t}^{T}\left(F^{T} F+A_{c l}^{T} S A_{c l}\right) x_{t}-x_{t+1}^{T} S x_{t+1} \\
& +r_{t}^{T}\left(I+B^{T} S B\right) r_{t} \\
& =x_{t}^{T} S x_{t}-x_{t+1}^{T} S x_{t+1}+r_{t}^{T}\left(I+B^{T} S B\right) r_{t}
\end{aligned}
$$

by using the equality $S=F^{T} F+A_{c l}^{T} S A_{c l}$ which is an other representation of the DARE in (6). Finally, substitute $J_{t}$ into $J$ and by using (26)

$$
\begin{aligned}
J= & \frac{1}{2} x_{t_{f}}^{T} S x_{t_{f}}+\frac{1}{2} \sum_{t=0}^{t_{f}-1} J_{t} \\
= & \frac{1}{2} x_{t_{f}}^{T} S x_{t_{f}}+\frac{1}{2} \sum_{t=0}^{t_{f}-1}\left(x_{t} S x_{t}-x_{t+1} S x_{t+1}\right) \\
& +\frac{1}{2} \sum_{t=0}^{t_{f}} r_{t}^{T}\left(I+B^{T} S B\right) r_{t} \\
= & \frac{1}{2} x_{t_{f}}^{T} S x_{t_{f}}+\frac{1}{2} \sum_{t=0}^{t_{f}-1}\left(x_{t}^{T} S x_{t}-x_{t+1}^{T} S x_{t+1}\right) \\
+ & \frac{1}{2} y_{f}^{T} Y_{c}\left(t_{f}\right)^{-1} y_{f} \\
= & \frac{1}{2} x_{0}^{T} S x_{0}+\frac{1}{2} y_{f}^{T} Y_{c}\left(t_{f}\right)^{-1} y_{f}=\frac{1}{2} y_{f}^{T} Y_{c}\left(t_{f}\right)^{-1} y_{f} .
\end{aligned}
$$

